\title{
Training with inspiratory pressure support in patients with severe COPD
}

\author{
A. van 't Hul*,\#, R. Gosselink ${ }^{\natural}$, P. Hollander ${ }^{\#,+}$, P. Postmus ${ }^{\S}$ and G. Kwakkel ${ }^{f, \#}$
}

ABSTRACT: This study evaluates the effects of training with noninvasive ventilatory support in patients with chronic obstructive pulmonary disease in a randomised, controlled, observerblinded trial.

Twenty-nine patients with chronic obstructive pulmonary disease and with a ventilatory limited exercise capacity (forced expiratory volume in one second $<60 \%$ predicted, breathing reserve at maximal exercise $<\mathbf{2 0} \%$ of maximally voluntary ventilation, resting arterial oxygen tension $\geqslant \mathbf{8} \mathrm{kPa}$ $(60 \mathrm{mmHg})$, end-exercise arterial oxygen saturation measured by pulse oximetry $\geqslant 85 \%$ ) completed an 8-week supervised outpatient cycle exercise programme. Fourteen patients were randomised to training with inspiratory pressure support of $10 \mathrm{cmH}_{2} \mathrm{O}$ and 15 patients to training with control (sham) inspiratory pressure support of $5 \mathrm{cmH}_{2} \mathrm{O}$. Outcome measures were the incremental shuttle walking test and a constant-load cycle endurance test at $75 \%$ of peak work rate including the measurement of physiological responses, and health status measured using the St. George's Respiratory Questionnaire.

Statistically significant between-group differences were found in favour of the inspiratory pressure support of $10 \mathrm{cmH}_{2} \mathrm{O}$ group for improvement in shuttle walking distance (16 \pm 17 versus $3 \pm 13 \%)$, cycle endurance (164 \pm 124 versus $88 \pm 128 \%$ ), and the reduction in minute ventilation during exercise $(-11 \pm 10$ versus $-2 \pm 9 \%)$.

It was concluded that exercise training with inspiratory pressure support of $10 \mathrm{cmH}_{2} \mathrm{O}$ resulted in statistically significantly larger improvements in exercise performance than training with inspiratory pressure support of $5 \mathrm{cmH}_{2} \mathrm{O}$ in patients with chronic obstructive pulmonary disease suffering from a ventilatory limited exercise capacity. Inspiratory pressure support of $10 \mathrm{cmH}_{2} \mathrm{O}$ may be considered as adjunct during high-intensity exercise training.

KEYWORDS: Assisted ventilation, chronic obstructive pulmonary disease, exercise, pulmonary rehabilitation

M eta-analysis has demonstrated that patients with chronic obstructive pulmonary disease (COPD) benefit significantly from rehabilitation programmes if exercise training is included [1, 2]. Furthermore, there are indications that more physiological benefit can be obtained in patients with COPD through highintensity training compared with moderateintensity training $[3,4]$. However, high-intensity exercise training may not be feasible to many patients [5]. Noninvasive ventilatory support (NIVS) has been recognised as an important adjunct during exercise, resulting in better exercise training tolerance in COPD [6].

To date, four randomised, controlled studies have compared the effects of training with NIVS to unsupported training in patients with COPD

For editorial comments see page 3 .
[7-10]. Two studies reported a between-group difference in the maximal incremental cycle exercise test in favour of patients training with NIVS, but not in the constant work rate cycle endurance test $[9,10]$. In contrast, JoHNSON et al. [7] found a significant between-group difference in the gain in walking endurance in favour of the group training with NIVS. Finally, BIANCHI et al. [8] observed no differential effect of training with NIVS on exercise performance or health status. All four studies, apart from having different outcomes, suffer from important methodological limitations. First, none incorporated a "sham" type of NIVS, contrasting the effects of training. Consequently, a placebo effect of NIVS could not be ruled out to explain the differences in effect. Secondly, observers were not blinded to treatment allocation in any of these studies. Absence of observer blinding may present a severe threat to the validity of
AFFILIATIONS

*Rehabilitation Centre Breda

${ }^{\S}$ Depts of Pulmonology and

${ }^{f}$ Physiotherapy VU University Medical Centre,

+Faculty of Human Movement Sciences, and

*Institute for Fundamental and Clinical Human Movement Science, Vrije Universiteit, Amsterdam, the Netherlands.

-Dept of Rehabilitation Sciences, Faculty of Kinesiology and Rehabilitation Sciences, Katholieke Universiteit Leuven, Belgium.

CORRESPONDENCE

A. van 't $\mathrm{Hul}$

Rehabilitation Centre Breda Brabantlaan 1 4817 JW Breda the Netherlands Fax: 31765331790 E-mail: a.vanthul@rcbreda.nl

Received:

March 272005

Accepted after revision:

September 222005

SUPPORT STATEMENT

This study was supported by a grant from the Dutch Asthma Foundation (grant 3.2.99.28) 
outcomes resulting in a significant overestimation of effect sizes [11]. Thirdly, the small number of patients included in these studies may have resulted in false negative decisions in accepting the $\mathrm{H}_{\mathrm{O}}$ null hypothesis due to lack of sufficient statistical power.

This randomised, controlled, observer-blinded, clinical trial set out to compare the effects of training with inspiratory pressure support of $10 \mathrm{cmH}_{2} \mathrm{O}$ (IPS10) to training with sham inspiratory pressure support of $5 \mathrm{cmH}_{2} \mathrm{O}$ (IPS5) on exercise performance and health status in patients with COPD and a ventilatory limited exercise capacity.

\section{METHODS}

\section{Study population}

Criteria for inclusion in the present study were: 1) diagnosis of COPD according to the criteria set by the Global Initiative for Chronic Obstructive Lung Disease (GOLD) scientific committee [12]; 2) forced expiratory volume in one second (FEV1) $<60 \%$ predicted; 3 ) a breathing reserve at maximal exercise $<20 \%$ of maximal voluntary ventilation (defined as $37.5 *$ FEV1) at peak exercise; 4) peak minute ventilation $<50 \mathrm{~L} \cdot \mathrm{min}^{-1}$; 5) resting arterial oxygen tension $>8 \mathrm{kPa}(60 \mathrm{mmHg})$; 6) pulse oximetry oxygen saturation at maximal exercise $\geqslant 85 \%$; 7 ) aged between 40 and 75 yrs; and 8) written informed consent. The study was approved by the Ethical Committee of the VU University Medical Centre, Amsterdam.

\section{Intervention}

Patients were randomly allocated (sealed envelopes) to training with IPS10 or $5 \mathrm{cmH}_{2} \mathrm{O}$ (IPS5). The exercise programme consisted of exercising on a calibrated cycle ergometer for 8 weeks, three times per week. Each treatment session lasted $45 \mathrm{~min}$, during which time patients were encouraged to exercise for as long as possible. For the first training session, training intensity was set at $65 \%$ of maximum work (Wmax). When, during this or a subsequent training session, a patient was able to exercise $>15 \mathrm{~min}$, the training intensity of the next session was increased by $5 \%$ of Wmax. During the study, other components of pulmonary rehabilitation, such as breathing retraining, education, relaxation or peripheral muscle resistance training, were not provided. Due to practical constraints, it was not possible to blind the three physiotherapists to the inspiratory pressure support (IPS) intensity patients were training with, but patients were kept naïve with respect to randomisation outcome. All measurements were performed by one independent investigator (A. van 't Hul), who was not involved in the training and who was kept blinded to randomisation outcome.

\section{Noninvasive ventilatory support}

Patients received IPS from a flow-triggered ventilator (Raphael; Hamilton AG, Thäzüns, Switzerland). The flow trigger was set at $3 \mathrm{~L} \cdot \mathrm{min}^{-1}$, i.e. the lowest possible value, and the inspiratory oxygen fraction at $21 \%$. This ventilator has a capacity to support minute ventilation up to $50 \mathrm{~L} \cdot \mathrm{min}^{-1}$ with a response time of $145 \mathrm{~ms}$ and a maximal inspiratory flow rate of $180 \mathrm{~L} \cdot \mathrm{min}^{-1}$. Patients were attached to the ventilator via corrugated tubing, and a mouthpiece and nose clip were applied. The dead space of the mouthpiece was $<15 \mathrm{~mL}$. Ventilator inspiratory and expiratory breathing circuits were separated, which prevented the possibility of rebreathing. On the basis of previous studies, IPS10 was considered adequate pressure support to increase exercise tolerance in COPD patients [13-15]. Exercise with IPS5 was considered appropriate control intervention because IPS5 during exercise appeared not to affect exercise endurance in patients with COPD [16].

\section{Baseline assessments}

Pulmonary function variables were measured (Vmax229; Sensormedics, Yorba Linda, CA, USA) according to the European Respiratory Society recommendations [17]. The method of BLACK and HYATT [18] was used for the measurement of maximal respiratory pressures. A symptom-limited incremental maximal cycle exercise test was conducted according to the European Respiratory Society recommendations [19]. The test was performed on a calibrated, electrically braked cycle ergometer (WLP 904; Lode, Groningen, the Netherlands) at a pedalling rate of 60 revolutions $\cdot \mathrm{min}^{-1}$. During testing, work rate was increased by $10 \%$ of the estimated maximal workload until exhaustion. Maximal heart rate was determined using the $\mathrm{R}-\mathrm{R}$ interval from a three-lead electrocardiogram (HP78351A; Hewlett Packard, Andover, MA, USA). Peak minute ventilation $\left(V^{\prime}\right.$ Epeak in $\mathrm{L} \cdot \mathrm{min}^{-1}$ at body temperature and ambient pressure, and saturated with water vapour) and peak oxygen uptake $\left(V^{\prime} \mathrm{O}_{2}\right.$ peak in $\mathrm{mL} \cdot \mathrm{min}^{-1}$ at standard temperature and pressure, and dry) were measured with a breath-by-breath automated exercise metabolic system (Vmax229). Arterial blood samples were drawn from an indwelling catheter in the radial artery at rest and at maximal exercise and analysed (Rapidlab®840; Bayer AG, Leverkusen, Germany). Wmax and $V^{\prime} \mathrm{O}_{2}$ peak were related to the reference of JONES and coworkers [20, 21]; $V^{\prime}$ Epeak was related to the predicted maximal voluntary ventilation [22]. At baseline, before the start of the training period, the acute effects of IPS10 and IPS5 were evaluated during exercise on constant work rate cycle endurance in all patients. Data on the acute responses to IPS during exercise in COPD of 21 patients were included in the current paper [16].

\section{Outcome measures}

Walking distance was measured using the incremental shuttle walking test, which was carried out twice at baseline and also at post-training. Results of the second tests were used in the analysis [23]. Symptom-limited exercise endurance was determined with a constant work rate cycle endurance test at $75 \%$ of the previously determined Wmax. End-exercise physiological responses from the baseline test were compared with posttraining responses at the isotime (the very moment that the baseline test was ended). The outcome of this endurance test has been shown to be highly reproducible in patients with COPD [24]. Health status was assessed using the St. George's Respiratory Questionnaire (SGRQ). The SGRQ is a self-report questionnaire and scores health status in three areas: symptoms (dyspnoea, wheezing and coughing), activity (the severity to which activities of daily living are impaired by dyspnoea) and impacts (the influence of respiratory symptoms on social participation) [25]. An improvement of more than $4 \%$ in total score (expressed as a percentage of the maximum score) is considered to be clinically relevant [26].

At baseline, all measurements were performed within 2 weeks prior to the start of the training period. Post-training 
measurements took place within 2 weeks after the final training session. All exercise tests were performed on separate days to avoid a sequential influence of test order. The assessor actually carrying out all measurements in the current study was not informed about randomisation outcome before all post-training assessments were completed in all patients.

\section{Statistical analysis}

Between-group differences were evaluated by means of independent-samples t-test or Mann-Whitney U-test. Withingroup differences were evaluated by means of a paired t-test or Wilcoxon signed ranks test. An intention-to-treat analysis was carried out assuming that patients who dropped out had the same change as the average improvement in the IPS5 group. Pearson's correlation coefficients were calculated to evaluate associations between variables. Values are expressed by their means and SD. For all tests a two-sided significance level of 0.05 was used.

\section{RESULTS}

A total of 37 patients entered the study; 18 were randomised to training with IPS5 and 19 to training with IPS10. One patient randomised to the IPS10 group dropped out after 4 weeks of training because of worsening general fatigue. In the IPS5 group, one patient dropped out because of a cerebrovascular accident. In both groups, three patients were lost due to acute exacerbation of COPD. Those who dropped out did not differ statistically significantly in pulmonary function or exercise performance from the patients completing the protocol.

\section{Study population}

Baseline physical characteristics, pulmonary function and maximal exercise performance of the patients completing the protocol are provided in tables 1 and 2. At baseline, no statistically significant between-group differences were present in age, stature, pulmonary function, exercise performance or health status. In the IPS10 group, six patients had a Medical Research Council (MRC) score of 3, and eight patients had a score of 4 . In the IPS5 group, five patients had an MRC score of 3 and 10 had a score of 4 . All patients received (inhalation) drug therapy; this was not changed during the study. None of the patients was familiar with NIVS before participation in this study and no patient was on home mechanical ventilation.

\section{Acute responses to IPS5 and IPS10}

In the 29 patients completing the protocol, exercise endurance time with IPS10 was statistically significantly higher compared with exercise without IPS ( $7.6 \pm 4.8$ versus $4.6 \pm 2.6 \mathrm{~min}$; $\mathrm{p}<0.001$ ), as well as compared with exercise with IPS5 ( $7.6 \pm 4.8$ versus $4.7 \pm 2.5 \mathrm{~min} ; \mathrm{p}<0.001)$. Endurance time did not differ significantly between tests without IPS and IPS5 $(4.6 \pm 2.6$ versus $4.7 \pm 2.5$ min; $\mathrm{p}=0.10)$.

\section{Effects on exercise training intensity}

In figure 1, average exercise intensity during training is depicted for both groups during the 8-week training period. Training intensities expressed as a percentage of the baseline Wmax differed statistically significantly between both groups in each week. During the first week, training intensity was increased, according to the protocol, in five patients in the group training with IPS10 (three patients at the first session and

\begin{tabular}{|c|c|c|}
\hline & NIVS group & Control group \\
\hline Patients n & 14 & 15 \\
\hline Age yrs & $70 \pm 5$ & $71 \pm 4$ \\
\hline Sex female/male & $4 / 10$ & $1 / 14$ \\
\hline $\mathrm{BMI} \mathbf{k g} \cdot \mathrm{m}^{-2}$ & $24.1 \pm 2.9$ & $24.4 \pm 3.5$ \\
\hline \multicolumn{3}{|l|}{ Pulmonary function } \\
\hline FEV 1 L & $1.06 \pm 0.36(41 \pm 10)$ & $1.10 \pm 0.24(38 \pm 9)$ \\
\hline FVC L & $3.01 \pm 0.90(87 \pm 14)$ & $2.84 \pm 0.45(76 \pm 14)$ \\
\hline $\mathrm{FEV}_{1} / \mathrm{FVC}$ & $0.34 \pm 0.06(45 \pm 8)$ & $0.36 \pm 0.08(49 \pm 10)$ \\
\hline$R V L$ & $3.79 \pm 0.66(161 \pm 18)$ & $4.17 \pm 1.25(164 \pm 50)$ \\
\hline $\mathrm{FRC} \mathrm{L}$ & $4.89 \pm 0.91(148 \pm 13)$ & $5.29 \pm 1.35(150 \pm 38)$ \\
\hline TLC L & $7.22 \pm 1.43(118 \pm 9)$ & $7.32 \pm 1.04(112 \pm 16)$ \\
\hline$D \mathrm{~L}, \mathrm{CO} \mathrm{mmol} \cdot \mathrm{kPa}^{-1} \cdot \mathrm{min}^{-1}$ & $4.29 \pm 1.58(50 \pm 19)$ & $3.68 \pm 1.36(44 \pm 17)$ \\
\hline $\mathrm{Pl}_{\mathrm{l} \text { max }} \mathrm{cmH}_{2} \mathrm{O}$ & $58 \pm 20(62 \pm 18)$ & $59 \pm 21(58 \pm 24)$ \\
\hline$P$ E,max $\mathrm{cmH}_{2} \mathrm{O}$ & $100 \pm 44(58 \pm 18)$ & $111 \pm 28(62 \pm 19)$ \\
\hline
\end{tabular}

Data are presented as mean \pm SD (\% predicted), except where otherwise stated. NIVS: noninvasive ventilatory support; BMI: body mass index; FEV1: forced expiratory volume in one second; FVC: forced vital capacity; RV: residual volume; FRC: functional residual capacity; TLC: total lung capacity; DL,CO: transfer factor for carbon monoxide; Pl,max: maximal inspiratory pressure; $P E$,max: maximal expiratory pressure.

TABLE 2 Incremental maximal exercise test

\begin{tabular}{|c|c|c|}
\hline & NIVS group & Control group \\
\hline Patients n & 14 & 15 \\
\hline Wmax watts & $56 \pm 26(46 \pm 19)$ & $58 \pm 21(40 \pm 14)$ \\
\hline$V^{\prime} O_{2}$, peak $L \cdot \min ^{-1}$ & $0.96 \pm 0.38(55 \pm 19)$ & $1.00 \pm 0.27(54 \pm 17)$ \\
\hline$V^{\prime} E$,peak $L^{\#}$ & $40.6 \pm 14.4(94 \pm 19)$ & $38.1 \pm 10.0(93 \pm 16)$ \\
\hline Heart rate maximum beats $\cdot \min ^{-1}$ & $122 \pm 20(82 \pm 13)$ & $117 \pm 16(79 \pm 11)$ \\
\hline \multicolumn{3}{|l|}{ Blood gases } \\
\hline $\mathrm{Pa}, \mathrm{O}_{2} \mathrm{kPa}$ rest/peak exercise & $9.8 \pm 1.2 / 8.3 \pm 1.8$ & $9.8 \pm 1.4 / 7.9 \pm 1.3$ \\
\hline $\mathrm{Pa}, \mathrm{CO}_{2} \mathrm{kPa}$ rest/peak exercise & $5.2 \pm 0.7 / 5.8 \pm 0.8$ & $5.4 \pm 0.8 / 5.7 \pm 0.7$ \\
\hline $\mathrm{Sa}, \mathrm{O}_{2} \%$ rest/peak exercise & $95 \pm 2 / 89 \pm 5$ & $95 \pm 2 / 88 \pm 5$ \\
\hline
\end{tabular}

Data are presented as mean \pm SD (\% predicted), except where otherwise indicated. \#: Data are presented as mean \pm SD (\% maximum voluntary ventilation). NIVS: noninvasive ventilatory support; Wmax: maximal workload; $V^{\prime} \mathrm{O}_{2}$,peak: peak pulmonary oxygen uptake; $V^{\prime} E$,peak: peak minute ventilation; $\mathrm{Pa}, \mathrm{O}_{2}$ : arterial oxygen tension; $\mathrm{Pa}_{\mathrm{a}} \mathrm{CO}_{2}$ : arterial carbon dioxide tension; $\mathrm{Sa}, \mathrm{O}_{2}$ : arterial oxyhaemoglobin saturation

another two at the second session) because they were able to exercise for longer than $15 \mathrm{~min}$ on the initial load $(65 \%$ of Wmax). In contrast, in the IPS5 group, only one patient was able to do so. During the first week of training, this already resulted in a significant between-group difference in average training work rate. In both groups, patients exercised effectively for $\sim 25$ min during each session. A moderate but significant correlation coefficient was found between the increase in work rate during the training period in the IPS10 group and the acute increase in cycle endurance resulting from the application of IPS10 measured at baseline $(r=0.45 ; \mathrm{p}=0.02)$. 


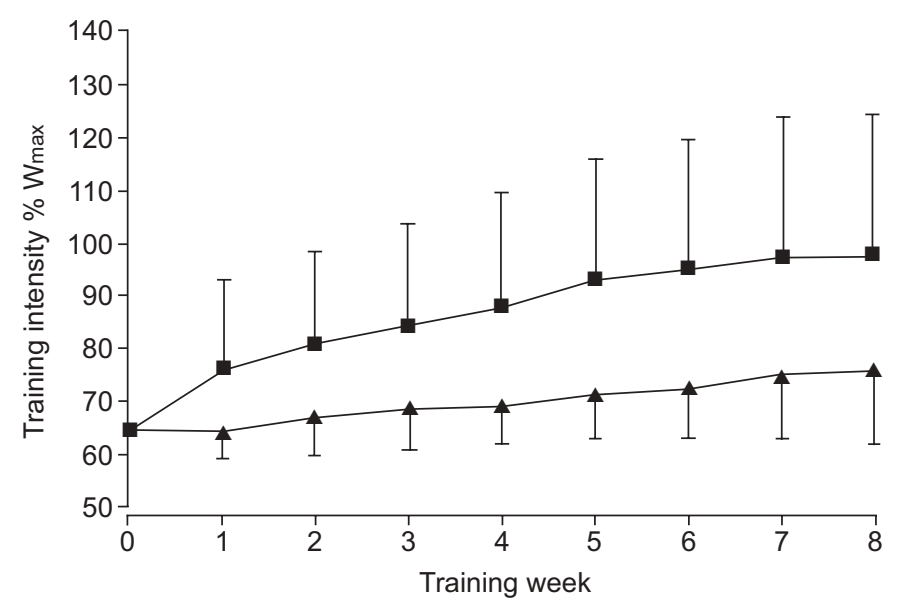

FIGURE 1. Training intensities expressed as percentage of maximal power output (Wmax) achieved during the incremental maximal cycle test at baseline. Values and error bars represent the mean and SD. In each week of training, patients trained with noninvasive ventilatory support in the form of inspiratory pressure support of $10 \mathrm{cmH}_{2} \mathrm{O}$ (ם) were able to train, on average, at significantly higher intensities $(\mathrm{p}<0.05)$ than patients trained with inspiratory pressure support of $5 \mathrm{cmH}_{2} \mathrm{O}(\boldsymbol{\Lambda})$. All patients started training at $65 \%$ of Wmax, but, during the first week, five patients in the group training with inspiratory pressure support of $10 \mathrm{cmH}_{2} \mathrm{O}$ were able to increase their training intensity. In contrast, in the group trained with sham inspiratory pressure support of $5 \mathrm{cmH}_{2} \mathrm{O}$, only one patient was able to do so. This resulted in a significant between-group difference in average training work rate during the first week of training.

\section{Effects of training on exercise performance}

The between-group difference in improvement in walking distance was statistically significant expressed either as a distance $(31 \pm 21$ versus $14 \pm 31 \mathrm{~m} ; \mathrm{p}<0.05)$ or as a percentage of the baseline test $(16 \pm 17$ versus $3 \pm 13 \%$; $p<0.05)$. Also, in the intention-to-treat analysis, the between-group difference remained statistically significant $(\mathrm{p}<0.05)$. A post hoc analysis revealed a statistical power of $66 \%$ to detect this difference in walking distance. Walking distance improved in 12 out of the 14 patients from the IPS10 group (on average from $253 \pm 132$ to $284 \pm 133 \mathrm{~m} ; \mathrm{p}<0.01)$ and in eight of the 15 patients from the IPS5 group (on average from $241 \pm 107$ to $255 \pm 128 ; p=0.10$ ).

The improvement in constant work rate cycle endurance time was larger in the IPS10 group compared with the IPS5 group ( $7.4 \pm 5.4$ versus $3.9 \pm 6.0 \mathrm{~min} ; \mathrm{p}=0.07)$, but only reached the level of significance when expressed as percentage improvement of baseline $(164 \pm 124$ versus $88 \pm 128 \%$; $<<0.05)$. In addition, in an intention-to-treat analysis, this between-group difference in improvement remained significant $(p<0.05)$. In the IPS10 group, 13 of the 14 patients improved (on average from $4.5 \pm 2.5$ to $9.9 \pm 6.1 \mathrm{~min} ; \mathrm{p}<0.001$ ), whereas, in the IPS5 group, 12 of the 15 patients increased their cycle endurance (on average from $4.5 \pm 2.8$ to $8.4 \pm 6.3 \mathrm{~min} ; \mathrm{p}<0.05$ ). Figures 2 and 3 show the improvements in incremental shuttle walking distance and cycle endurance, respectively, in both groups.

Table 3 shows the results of the physiological responses during the constant work-rate cycle endurance test. Data of endexercise responses during the baseline test and at isotime of the post-training test are provided. A statistically significant

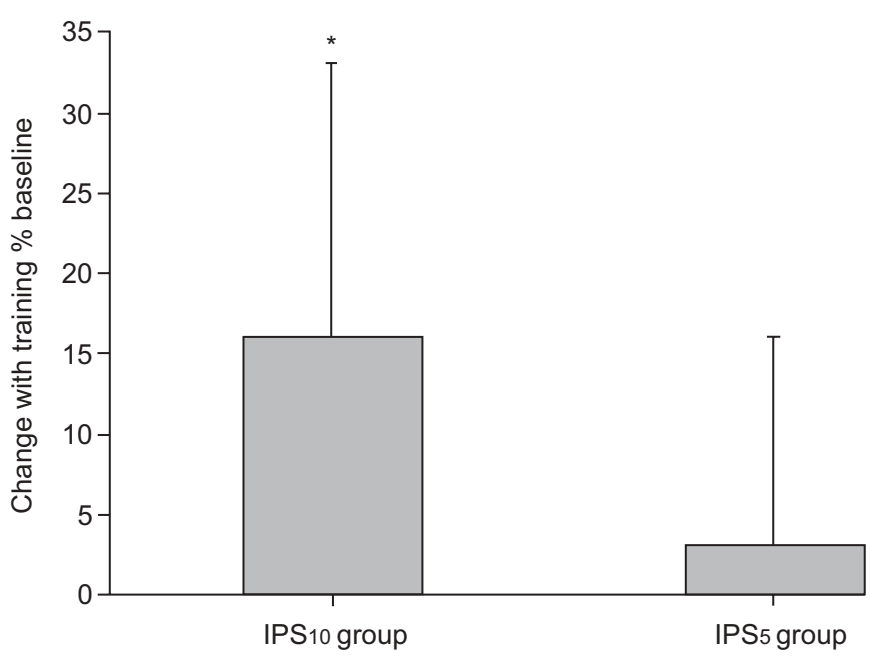

FIGURE 2. Change in incremental shuttle walking distance with training. * $p<0.05$.

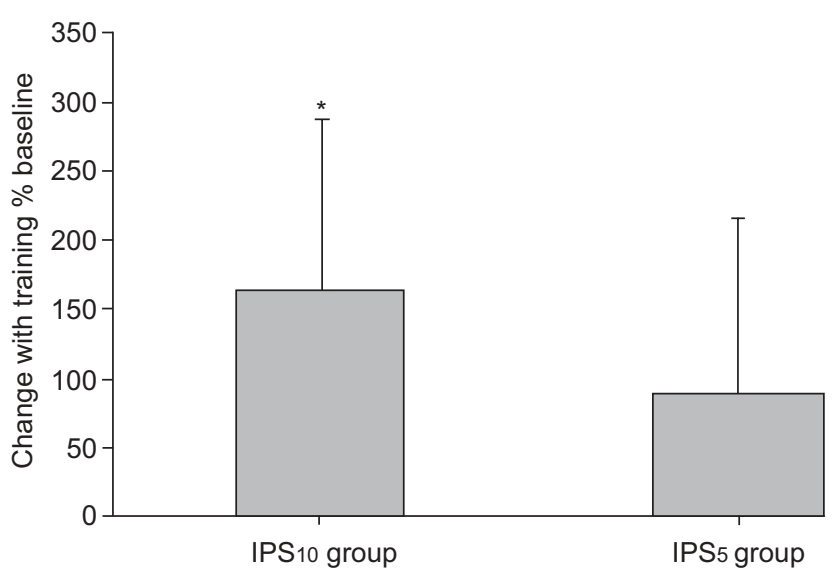

FIGURE 3. Change in constant-load cycle endurance with training. *: $p<0.05$.

between-group difference was found for the reduction in minute ventilation $\left(-4.0 \pm 3.2\right.$ versus $\left.-0.7 \pm 2.9 \mathrm{~L} \cdot \mathrm{min}^{-1} ; \mathrm{p}=0.01\right)$. Also, in the intention-to-treat analysis, this between-group difference in reduction in minute ventilation was statistically significant $(\mathrm{p}<0.05)$. In contrast to the IPS5 group, in the IPS10 group, significantly lower values were found for minute ventilation, respiratory rate, oxygen uptake and carbon dioxide output after training $(\mathrm{p}<0.05)$.

\section{Effects of training on health status}

Table 4 shows the results of the SGRQ. No significant betweengroup differences were observed at baseline. After training, a significantly lower score in the activity component was only observed in the IPS10 group. No significant between-group difference was observed after training in any component of the questionnaire.

\section{DISCUSSION}

This is the first randomised, controlled, observer-blinded trial in which outcomes of training with IPS10 were compared with training with IPS5 in patients with severe COPD. A statistically 
TABLE 3 Physiological responses during the constant-load cycle exercise test at end exercise of the baseline test and the corresponding moment (isotime) of the post-training test

NIVS group

Baseline end-exercise Post-training isotime
Control group

Baseline end-exercise Post-training isotime

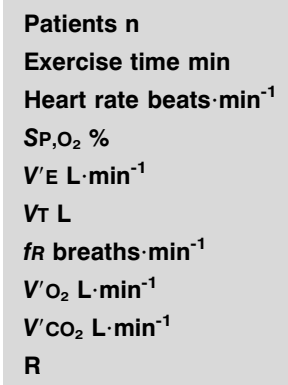

14

$$
\begin{gathered}
4.5 \pm 2.5 \\
113 \pm 18 \\
91 \pm 5 \\
33.6 \pm 12.9 \\
1.13 \pm 0.35 \\
29 \pm 5 \\
0.77 \pm 0.27 \\
0.72 \pm 0.28 \\
0.93 \pm 0.06
\end{gathered}
$$

$4.5 \pm 2.5$
$107 \pm 10^{\#}$
$91 \pm 5$
$29.6 \pm 11.7^{\#}$
$1.22 \pm 0.36$
$24 \pm 5^{\circ}$
$0.70 \pm 0.23^{\circ}$
$0.64 \pm 0.24^{\circ}$
$0.91 \pm 0.07$

15

$$
\begin{gathered}
4.6 \pm 2.8 \\
112 \pm 16 \\
90 \pm 4 \\
33.7 \pm 7.8 \\
1.24 \pm 0.35 \\
28 \pm 6 \\
0.78 \pm 0.19 \\
0.72 \pm 0.18 \\
0.93 \pm 0.07
\end{gathered}
$$

\begin{tabular}{|c|c|c|c|c|}
\hline & Baseline & Post-training & Baseline & Post-training \\
\hline Patients n & \multicolumn{2}{|c|}{14} & \multicolumn{2}{|c|}{15} \\
\hline Symptoms & $338 \pm 131(50 \pm 19)$ & $296 \pm 136(45 \pm 20)$ & $343 \pm 139(52 \pm 21)$ & $297 \pm 151(47 \pm 24)$ \\
\hline Activities & $913 \pm 234(76 \pm 19)$ & $814 \pm 188(67 \pm 15)^{\#}$ & $992 \pm 150(82 \pm 12)$ & $912 \pm 193(75 \pm 16)$ \\
\hline
\end{tabular}

$4.6 \pm 2.8$

$104 \pm 12^{\#}$

$89 \pm 5$

$31.6 \pm 6.4$

$1.20 \pm 0.36$

$27 \pm 6$

$0.74 \pm 0.17$

$0.67 \pm 0.16$

$0.91 \pm 0.06$

Data are presented as mean \pm SD. NIVS: noninvasive ventilatory support; $S P, \mathrm{O}_{2}$ : oxyhaemoglobin saturation measured with pulse oximetry; $V^{\prime} E$ : minute ventilation; VT: tidal volume; $f R$ : respiratory frequency; $V^{\prime} \mathrm{O}_{2}$ : oxygen uptake; $V^{\prime} \mathrm{CO}_{2}$ : carbon dioxide production; $\mathrm{R}$ : respiratory exchange ratio. ${ }^{\#}$ : statistically significantly different from pretraining, $p<0.05 ;{ }^{\bullet}$ : statistically significantly different from pre-training, $p<0.01$.

\section{TABLE 4 St. George's Respiratory Questionnaire}

Data are presented as mean \pm SD (\%). NIVS: noninvasive ventilatory support. \#: statistically significantly different from pre-training, $p<0.05$.

significant between-group difference was found in the training intensity during the training period expressed as a percentage of the baseline's Wmax, and was in favour of patients training with IPS10. In addition, significant between-group differences were found in improvement in the incremental shuttle walking test and improvement in cycle endurance at $75 \%$ of maximal power output, as well as in the reduction in minute ventilation during the cycle endurance test. Changes in health status measured with the SGRQ did not differ significantly between both groups.

\section{Patient selection}

Patients were selected for the current study on the basis of the existence of a significant ventilatory impaired exercise capacity without marked pulmonary oxygen transport deficit. This was based on the authors' assumption that the application of IPS10 might especially benefit this subgroup of patients. Randomisation resulted in well-balanced groups with respect to possible prognostic factors for outcome of exercise training, such as disease severity and maximal exercise parameters. In addition, the included patients had a marked inspiratory muscle weakness. Since weakness makes these muscles susceptible to fatigue this might have contributed to the positive outcome of training with IPS10 in the present study [27].

\section{Interpretation of the effects of training with IPS10}

As observed by HAWKINS et al. [9], it was found that patients training with IPS10 were able to achieve significantly higher training intensities throughout the 8-week training period. In the last week of training, intensities were $76 \pm 14$ and $94 \pm 27 \%$ of the baseline's Wmax, in the IPS5 and the IPS10 group, respectively. These intensities are considerably higher compared with the maximal tolerable exercise intensity during an 8-week training programme in a study by MALTAIs et al. [5], who found a mean training intensity of $60 \%$ during the final week. Recently, EMTNER et al. [28] reported exercise intensities up to $96 \%$ of baseline's Wmax in patients with a similar degree of airway obstruction, training on room air. These betweenstudy differences in maximal tolerable exercise intensity are not readily explained, but may be due to a variation in study populations, differences in the amount of encouragement by the supervisors of the training, or the way Wmax was determined [29]. 
In the group training with IPS10, a two-fold larger increase in constant work rate cycle endurance was found. Unfortunately, no data are available with respect to the minimal change required to represent a clinically meaningful change. However, the increase in cycle endurance appeared to be higher than in other studies of COPD patients with a similar airway obstruction receiving training on a cycle ergometer without NIVS [4,30], and was also considerably higher than the effect of a long-acting bronchodilator on exercise endurance [31]. Similar to the results of the current study are the greater improvements in cycle endurance obtained with training with NIVS in the studies of HAWKINS et al. [9] and COSTES et al. [10]. By contrast, BIANCHI et al. [8] did not observe a significant differential effect in either training intensity or exercise performance between COPD patients training with and without proportional assist ventilation (PAV). Possible explanations for the contrasting findings between Bianchi's study and the current and other studies [7, 9-10] are patient characteristics, such as the relatively mild ventilatory impairment and the relatively preserved exercise performance (Wmax $=85 \mathrm{watt})$. Furthermore, the intolerance for NIVS during exercise training in Bianchi's study might have resulted from the use of a nasal mask instead of a full-face mask or mouthpiece. During strenuous exercise, patients may prefer to breathe through their mouth rather than through their nose.

Dyspnoea scores were not rated during exercise in the current study. Despite the reported reliability of exertional dyspnoea measurement by some, it is the experience of the authors, as well as of others, that measures to rate symptoms (Borg scores, visual analogue scales) may vary to a great extent within individuals during similar exercise loads and duration on repeated testing. In addition, it was anticipated that ratings were impractical during exercise with NIVS because of difficulties with verbal communications, acknowledging that ratings of dyspnoea may interfere with patients' necessary concentration to exercise with NIVS. The reduction in minute ventilation at identical work rate and/or duration may improve exercise performance in COPD, because of the reduction in dynamic hyperinflation [32]. This reduction might be induced by the lower respiratory rate found in the IPS10 group. Interestingly, the effects of training with IPS10 on breathing pattern, that is, a reduction in respiratory rate, show great similarities with the acute effects of IPS10 $[13,16]$. This suggests that training with IPS10 may induce a breathing retraining effect. Alternatively, training with IPS10 might have resulted in a superior improvement in lower limb muscle function compared with training with IPS5. Unfortunately, muscle function tests were not included in the assessment protocol of the current study.

\section{Noninvasive ventilatory support type and settings}

Different modalities of NIVS have been used successfully to improve exercise tolerance acutely in patients with COPD, such as continuous positive airway pressure, IPS and PAV, either in combination with positive end expiratory pressure or not. In addition, marked differences in pressure intensities have been used. In a systematic review on the acute effects of NIVS in patients with COPD, no significant heterogeneity was observed between the effect sizes of these modalities [6]. This suggests equal efficacy of continuous positive airway pressure,
IPS and PAV, in improving exercise training tolerance. In the studies reporting positive effects of training with NIVS in COPD patients, two studies used IPS [7, 10], and in one study PAV was used [9]. In the present study, the effects of training with two fixed levels of IPS, that is 5 and $10 \mathrm{cmH}_{2} \mathrm{O}$, were contrasted. It was decided the IPS intensities would not be individualised for methodological reasons, i.e. standardisation of the intervention. It was also anticipated that it would not be feasible to titrate individual inspiratory pressure or positive end expiratory pressure settings in the absence of the possibility to measure actual respiratory mechanics. IPS5 was anticipated to represent an adequate control intervention because it was found not to affect exercise endurance significantly in patients with COPD, despite a small improvement in breathing efficiency [16]. Also, in the patients participating in the present study, no difference was observed between exercise endurance with IPS5 compared with exercise without IPS. Future research may be directed at the effect of individualised inspiratory pressure settings and/or the addition of positive end expiratory pressure on the efficacy of NIVS during exercise training.

\section{Limitations of the study}

Obviously, the present study has limitations. First, the main methodological shortcoming of the present study is the lack of ability to blind the physiotherapists who provided the training to the treatment allocation. The resulting expectation bias may have resulted in differences in the extent to which patients were encouraged by the therapists during the exercise training. The amount of encouragement is likely to be a factor influencing a patient's motivation to train harder. However, to minimise this influence, physiotherapists were not informed about the acute effects of IPS10 at baseline in individual patients. Moreover, the exercise protocol was standardised and strictly adhered to. Secondly, differential effects of training with NIVS directly after the training episode were evaluated. There was no follow-up. No data were available in the literature on differential effects of training with NIVS at the time when the authors were preparing their study. In addition, after the training period, patients were offered continuous care, including additional training interventions and educational sessions. This was offered on the basis of individual need and thus varied between individuals. Thirdly, a significantly lower value of the SGRQ activity component was observed only in the patients trained with IPS10; no between-group difference was found. Apparently, the extra gain in exercise performance following training with IPS10 produced no additional gain in perceived health status. This finding confirms that changes in exercise performance and quality of life resulting from rehabilitation are independent [1]. Alternatively, the present study may be underpowered to detect statistically significant between-group differences in change due to training. Finally, the improvement in walking distance appeared to be low in this study. It must be noted, however, that patients were offered aerobic (cycle) exercise training as a monotherapy. Specificity of training might be another possible explanation, i.e. training on a cycle is likely to increase cycle endurance rather than walking distance. Also, other important components of pulmonary rehabilitation potentially contributing to improvements in health status, such as peripheral and respiratory muscle strength training, 
education and psychosocial support, were not provided. Therefore, if exercise training with NIVS is considered, the authors recommend combining it with other rehabilitation components, based on the individual needs, using the potential synergistic effects $[33,34]$.

\section{Clinical implications}

Practical drawbacks may accompany the use of NIVS as an adjunct during training. First, NIVS is a labour-intensive type of intervention, because of the required one-to-one patienttherapist ratio, and will add to the cost of rehabilitation programmes. Secondly, discomfort might be associated with the use of a mouthpiece or mask during NIVS. Although, in the present study, no patients dropped out on these grounds, the authors are aware that, in another study, a substantial number of patients was lost for this reason [8]. During the recruiting phase of the present study, the authors only came across a few patients who, after a trial session, resigned from further participation in the study because of discomfort related to the application of the NIVS. Again, this may be due to the patients that were selected for this study, i.e. patients with a clear ventilatory impaired exercise capacity.

Despite the selection of patients on the basis of a ventilatory impaired exercise capacity, there was still a large variability found in the extent to which patients were able to benefit from training with IPS10. In the current study, an association was observed between the increase in training intensity during the training period and the acute increase in training tolerance at baseline as a result of the application of IPS10. This indicates that patients who showed markedly acute improved exercise tolerance on IPS10 also tended to benefit most from training with IPS10. Regrettably, the predictive validity of the acute response was low. Consequently, the authors suggest that a single trial of exercise performance on IPS10 can be used to identify patients who might benefit from this intervention. Subsequently, training with IPS10 could be offered to patients unable to tolerate a high training intensity and in whom the application of IPS10 results in a significant increase in exercise tolerance.

In summary, the current study demonstrates the additional effects of training with inspiratory pressure support of 10 $\mathrm{cm} \mathrm{H}_{2} \mathrm{O}$ on exercise training intensity, constant work rate cycle endurance and shuttle walking distance in patients with chronic obstructive pulmonary disease and a ventilatory limited exercise capacity. Inspiratory pressure support of 10 $\mathrm{cmH}_{2} \mathrm{O}$ may be considered as an adjunct during exercise training aimed at high-intensity training, yielding greater improvements in exercise performance.

\section{ACKNOWLEDGEMENTS}

S. Hania, K. Huitenga and D. Raams, physiotherapists at the Department of Physiotherapy, VU University Medical Centre, Amsterdam, are gratefully acknowledged for their coaching of patients during their exercise training programme. R. Straver is thanked for her contribution during the writing of this paper.

\section{REFERENCES}

1 Lacasse Y, Brossea L, Milne S, et al. Pulmonary rehabilitation for chronic obstructive pulmonary disease. Cochrane Database Syst Rev 2002; 3: CD003793.
2 Lacasse Y, Guyatt GH, Goldstein RS. The components of a respiratory rehabilitation program: a systematic overview. Chest 1997; 111: 1077-1088.

3 Casaburi R, Patessio A, Ioli F, Zanaboni S, Donner CF. Reductions in exercise lactic acidosis and ventilation as a result of exercise training in patients with obstructive lung disease. Am Rev Respir Dis 1991; 143: 9-18.

4 Casaburi R, Porszasz J, Burns MR, et al. Physiologic benefits of exercise training in rehabilitation of patients with severe chronic obstructive pulmonary disease. Am J Respir Crit Care Med 1997; 155: 1541-1551.

5 Maltais F, LeBlanc P, Jobin J, et al. Intensity of training and physiologic adaptation in patients with chronic obstructive pulmonary disease. Am J Respir Crit Care Med 1997; 155: 555-561.

6 van 't Hul AJ, Kwakkel G, Gosselink R. The acute effects of noninvasive ventilatory support during exercise on exercise endurance and dyspnea in patients with chronic obstructive pulmonary disease: a systematic review. J Cardiopulm Rehabil 2002; 22: 290-297.

7 Johnson JE, Gavin DJ, Adams-Dramiga S. Effects of training with heliox and noninvasive positive pressure ventilation on exercise ability in patients with severe COPD. Chest 2002; 122: 464-472.

8 Bianchi L, Foglio K, Porta R, Baiardi R, Vitacca M, Ambrosino N. Lack of additional effect of adjunct of assisted ventilation to pulmonary rehabilitation in mild COPD patients. Respir Med 2002; 96: 359-367.

9 Hawkins P, Johnson LC, Nikoletou D, et al. Proportional assist ventilation as an aid to exercise training in severe chronic obstructive pulmonary disease. Thorax 2002; 57: 853-859.

10 Costes F, Agresti A, Court-Fortune I, Roche F, Vergnon JM, Barthelemy JC. Noninvasive ventilation during exercise training improves exercise tolerance in patients with chronic obstructive pulmonary disease. J Cardiopulm Rehabil 2003; 23: 307-313.

11 Moher D, Pham B, Jones A, et al. Does quality of reports of randomised trials affect estimates of intervention efficacy reported in meta-analyses? Lancet 1998; 352: 609-613.

12 Pauwels RA, Buist AS, Calverley PM, et al. Global strategy for the diagnosis, management, and prevention of chronic obstructive pulmonary disease. NHLBI/WHO Global Initiative for Chronic Obstructive Lung Disease (GOLD) Workshop summary. Am J Respir Crit Care Med 2001; 163: 1256-1276.

13 Maltais F, Reissmann H, Gottfried SB. Pressure support reduces inspiratory effort and dyspnea during exercise in chronic airflow obstruction. Am J Respir Crit Care Med 1995; 151: 1027-1033.

14 Bianchi L, Foglio K, Pagani M, Vitacca M, Rossi A, Ambrosino N. Effects of proportional assist ventilation on exercise tolerance in COPD patients with chronic hypercapnia. Eur Respir J 1998; 11: 422-427.

15 Kyroussis D, Polkey MI, Hamnegard CH, Mills GH, Green M, Moxham J. Respiratory muscle activity in patients with COPD walking to exhaustion with and without pressure support. Eur Respir J 2000; 15: 649-655.

16 van 't Hul AJ, Gosselink R, Hollander AP, Postmus PE, Kwakkel G. Acute effects of inspiratory pressure support 
during exercise in patients with COPD. Eur Respir J 2004; 23: 34-40.

17 Quanjer PH, Tammeling GJ, Cotes JE, Pedersen OF, Peslin R, Yernault JC. Lung volumes and forced ventilatory flows. Report Working Party Standardization of Lung Function Tests, European Community for Steel and Coal. Official Statement of the European Respiratory Society. Eur Respir J 1993; 16: Suppl. 3, 5-40.

18 Black LF, Hyatt RE. Maximal respiratory pressures: normal values and relationship to age and sex. Am Rev Respir Dis 1969; 99: 696-702.

19 Roca J, Whipp BJ, Agusti AN, et al. Clinical exercise testing with reference to lung diseases - indications, standardization and interpretation strategies. Eur Respir J 1997; 10: 2662-2689.

20 Jones NL, Makrides L, Hitchcock C, Chypchar T, Mc Cartney. Normal standards for an incremental progressive cycle ergometer test. Am Rev Respir Dis 1985; 131: 700-708.

21 Jones NL, Summers E, Killian KJ. Influence of age and stature on exercise capacity during incremental cycle ergometry in men and women. Am Rev Respir Dis 1989; 140: 1373-1380.

22 Carter R, Peavler M, Zinkgraf S, Williams J, Fields S. Predicting maximal exercise ventilation in patients with chronic obstructive pulmonary disease. Chest 1987; 92: 253-259.

23 Singh SJ, Morgan MD, Scott S, Walters D, Hardman AE. Development of a shuttle walking test of disability in patients with chronic airways obstruction. Thorax 1992; 47: 1019-1024.

24 van 't Hul AJ, Gosselink R, Kwakkel G. Constant-load cycle endurance performance: test-retest reliability and validity in patients with COPD. J Cardiopulm Rehabil 2003; 23: 143-150.

25 Jones PW, Quirk FH, Baveystock CM, Littlejohns P. A selfcomplete measure of health status for chronic airflow limitation. The St. George's Respiratory Questionnaire. Am Rev Respir Dis 1992; 145: 1321-1327.

26 Schunemann HJ, Griffith L, Jaeschke R, Goldstein R, Stubbing D, Guyatt GH. Evaluation of the minimal important difference for the feeling thermometer and the St. George's Respiratory Questionnaire in patients with chronic airflow obstruction. J Clin Epidemiol 2003; 56: 1170-1176.

27 Polkey MI, Kyroussis D, Mills GH, Somfay A, Casaburi R. Inspiratory pressure support reduces slowing of inspiratory muscle relaxation rate during exhaustive treadmill walking in severe COPD. Am J Respir Crit Care Med 1996; 154: 1146-1150.

28 Emtner M, Porszasz J, Burns M, Somfay A, Casaburi R. Benefits of supplemental oxygen in exercise training in nonhypoxemic chronic obstructive pulmonary disease patients. Am J Respir Crit Care Med 2003; 168: 1034-1042.

29 Debigare R, Maltais F, Mallet M, Casaburi R, LeBlanc P. Influence of work rate incremental rate on the exercise responses in patients with COPD. Med Sci Sports Exerc 2000; 32: 1365-1368.

30 Puente-Maestu L, Tena T, Trascasa C, et al. Training improves muscle oxidative capacity and oxygenation recovery kinetics in patients with chronic obstructive pulmonary disease. Eur J Appl Physiol 2003; 88: 580-587.

31 O'Donnell DE, Fluge $\mathrm{T}$, Gerken F, et al. Effects of tiotropium on lung hyperinflation, dyspnoea and exercise tolerance in COPD. Eur Respir J 2004; 23: 832-840.

32 O'Donnell DE, Revill SM, Webb KA. Dynamic hyperinflation and exercise intolerance in chronic obstructive pulmonary disease. Am J Respir Crit Care Med 2001; 164: 770-777.

33 Anonymas. Pulmonary rehabilitation. Thorax 2001; 56: 827-834.

34 O'Donnell DE, Aaron S, Bourbeau J, et al. Canadian Thoracic Society recommendations for management of chronic obstructive pulmonary disease - 2003. Can Respir J 2003; 10: Suppl. A, 11A-65A. 